it is likely that both preexisting mature hepatocytes and Sox $9^{+}$cells contribute to regeneration. One possibility is that the "quiescent" liver in the Sox9 knockin mice (17) is actually undergoing chronic injury, either via decreased Sox9 levels or toxicity due to tamoxifen or chronic CreERT2 expression. However, this would have to cause a non-cell autonomous inhibition of hepatocyte replication to result in the sort of oval/progenitor cell-derived repopulation of the liver seen in the DDC diet. By using a conditional lineage-tracing model that does not produce any measurable toxicity or liver injury, Malato and colleagues (1) provide strong evidence that it is hepatocytes themselves, and not cholangiocytes or specialized progenitor cells, that are responsible for the homeostatic renewal of the liver under basal and even mild injury conditions.

\section{Acknowledgments}

Related work in K.H. Kaestner's lab is supported by P01 DK049210 and in J.R. Friedman's lab by R01 DK079881. We thank our colleagues in the liver field for many stimulating discussions on the subject.
Address correspondence to: Klaus $\mathrm{H}$. Kaestner, University of Pennsylvania, 560A CRB/6140, Philadelphia, Pennsylvania, USA. Phone: 215.898.8759; Fax: 215.573.5892; E-mail: kaestner@mail.med.upenn.edu.

1. Malato Y, et al. Fate tracing of mature hepatocytes in mouse liver homeostasis and regeneration. JClin Invest. 2011;121(12):4850-4860.

2. Zorn AM. Liver development. In: Girard L, ed. StemBook. Cambridge, Massachusetts, USA: Harvard Stem Cell Institute; 2008.

3. Brues AM, Marble BB. An analysis of mitosis in liver restoration. J Exp Med. 1937;65(1):15-27.

4. Duncan AW, Dorrell C, Grompe M. Stem cells and liver regeneration. Gastroenterology. 2009;137(2):466-481.

5. Overturf K, al-Dhalimy M, Ou CN, Finegold M, Grompe M. Serial transplantation reveals the stem-cell-like regenerative potential of adult mouse hepatocytes. Am J Path. 1997;151(5):1273-1280.

6. Grisham JW. A morphologic study of deoxyribonucleic acid synthesis and cell proliferation in regenerating rat liver; autoradiography with thymidineH3. Cancer Res. 1962;22:842-849.

7. Zajicek G, Oren R, Weinreb M Jr. The streaming liver. Liver. 1985;5(6):293-300

8. Fletcher J, et al. The inhibitory role of stromal cell mesenchyme on human embryonic stem cell hepatocyte differentiation is overcome by Wnt3a treatment. Cloning Stem Cells. 2008; 10(3):331-339.

9. Kennedy S, Rettinger S, Flye MW, Ponder KP. Experiments in transgenic mice show that hepatocytes are the source for postnatal liver growth and do not stream. Hepatology. 1995;22(1):160-168.

10. Bralet MP, Branchereau S, Brechot C, Ferry N. Cell lineage study in the liver using retroviral mediated gene transfer. Evidence against the streaming of hepatocytes in normal liver. Am J Pathol. 1994;144(5):896-905.

11. Grisham JW. Grisham: reply. Am J Pathol. 1995; 146(3):773-776.

12. Suzuki A, et al. Flow cytometric isolation and clonal identification of self-renewing bipotent hepatic progenitor cells in adult mouse liver. Hepatology. 2008;48(6):1964-1978.

13. Wang X, Foster M, Al-Dhalimy M, Lagasse E, Finegold M, Grompe M. The origin and liver repopulating capacity of murine oval cells. Proc Natl Acad Sci US A. 2003;100 suppl 1:11881-11888.

14. Shin S, et al. Foxl1-Cre-marked adult hepatic progenitors have clonogenic and bilineage differentiation potential. Genes Dev. 2011;25(11):1185-1192.

15. Schwartz RE, et al. Multipotent adult progenitor cells from bone marrow differentiate into functional hepatocyte-like cells. J Clin Invest. 2002;109(10):1291-1302.

16. Jiang $\mathrm{Y}$, et al. Pluripotency of mesenchymal stem cells derived from adult marrow. Nature. 2002; 418(6893):41-49.

17. Furuyama K, et al. Continuous cell supply from a Sox9-expressing progenitor zone in adult liver, exocrine pancreas and intestine. Nat Genet. 2011; 43(1):34-41.

18. Antoniou A, et al. Intrahepatic bile ducts develop according to a new mode of tubulogenesis regulated by the transcription factor SOX9. Gastroenterology. 2009;136(7):2325-2333.

19. Seymour PA, et al. SOX9 is required for maintenance of the pancreatic progenitor cell pool. Proc Natl Acad Sci U S A. 2007;104(6):1865-1870.

\title{
Perception of sound and gravity by TMC1 and TMC2
}

Xi Lin

Departments of Otolaryngology and Cell Biology, Emory University School of Medicine, Atlanta, Georgia, USA.

\begin{abstract}
Central to our ability to hear and sense gravity is a cellular process known as mechanotransduction, which is initiated by the opening of mechanosensitive cation channels located near the tips of the stereocilia of auditory and vestibular inner ear hair cells. The molecular identity of the mechanotransduction channels has eluded researchers despite intensive investigations over the years. In this issue of the JCI, Kawashima et al. report their results obtained using mice with targeted deletion of both transmembrane channel-like 1 (Tmc1) and $T m c 2$. The use of inner ear hair cells isolated from these mice provided a nearly perfect system for testing the mechanotransduction channels without disrupting functions of other accessory proteins needed in the complicated molecular apparatus, and it allowed the authors to show that the proteins encoded by these genes are integral components of the mechanotransduction complex.
\end{abstract}

Auditory and vestibular inner ear hair cells are central to our ability to perceive sound

Conflict of interest: The author has declared that no conflict of interest exists.

Citation for this article: J Clin Invest. 2011; 121(12):4633-4636. doi:10.1172/JCI61167. and gravity, respectively. These cells convert mechanical stimuli initiated by sounds and gravitational force into electrical signals, a process known as mechanotransduction. Mechanotransduction is initiated when the mechanical stimuli cause stereocilia at the apical surface of the hair cells to move, triggering the opening of mechanosensitive cation channels located at the tip. Ionic currents that flow through the open channels initiate graded receptor potentials (refs. 1, 2, and Figure 1). This results in rapid changes in the membrane potential of the hair cells and subsequently modulates neurotransmitter release at the hair cell base. Auditory and vestibular inner ear hair cells synapse with auditory and vestibular nerves, respectively. Thus, changes in neurotransmitter release by the hair cells modulate postsynaptic action potentials to convey the mechanical information to the brain. These neuronal activities underlie our perception of sound and are necessary for maintaining balance and coordination of eye and head movements.

Molecular identification of the mechanotransduction channels in the stereocilia 

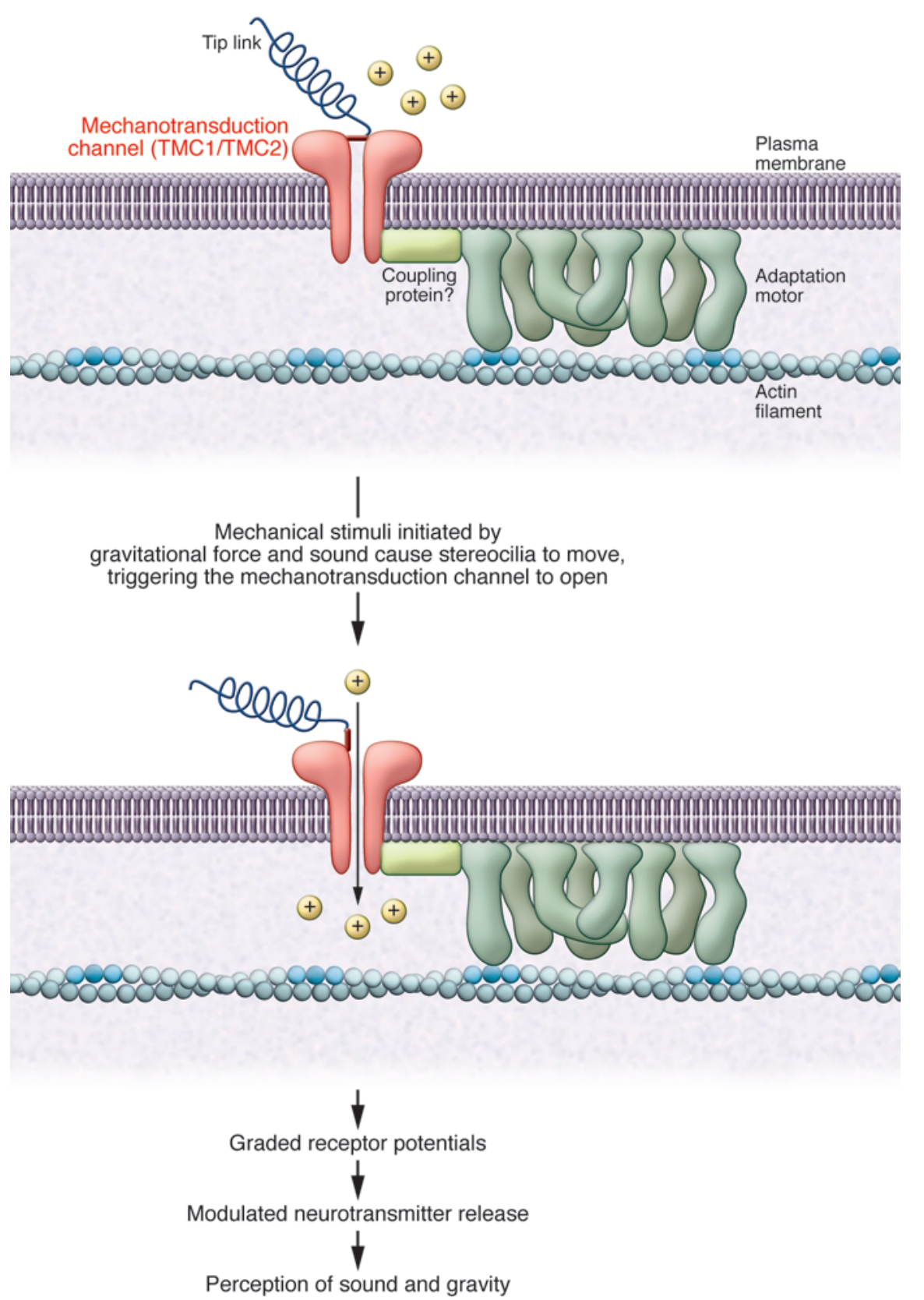

Figure 1

The mechanotransduction process in mammalian hair cells. Displacement of the stereocilia creates tension in tip links that open the mechanotransduction channels located near the tips of stereocilia. The mechanotransduction channel is coupled to an adaptation motor so tension of the tip link can be properly adjusted.

of auditory and vestibular hair cells is fundamental for enhancing our understanding of mammalian auditory and vestibular systems, and has been a major focus of research in this field for many years. However, whereas the molecular mechanisms of transduction in the visual system are well understood (3), the molecular identity of the components of the auditory and ves- tibular mechanotransduction apparatus in mammals is largely unknown. Many research groups around the world have been searching for answers, and in this issue of the JCI, Kawashima and colleagues present an intriguing possibility (4). Specifically, they uncover key roles for transmembrane channel-like 1 (TMC1) and TMC2 in hair cell mechanotransduction in mice that indicate that these proteins are likely to be integral molecular components of the auditory and vestibular mechanotransduction apparatus.

\section{Clues to the identity of the mechanotransduction channels}

Over the years, several proteins have been proposed as candidate mechanotransduction channels, with most suggestions being based on studies in lower animals. At one time, a mammalian equivalent of bacterial large-conductance mechanosensitive channels such as MscL was considered a reasonable candidate (5). Cyclic nucleotide-gated (CNG) channels in hair cells (6) have also been prime contenders. However, the rapid dynamic changes required for auditory transduction are in the sub-millisecond range for highfrequency sensing, making such a proposal unlikely to be true (3). Members of the degenerin/epithelial $\mathrm{Na}^{+}$channel (DEG/ENaC) family of ion channels have also been candidates, as they have been shown to be the major mechanoelectrical transduction channels in a Caenorbabditis elegans nociceptor (7). However, data obtained from transgenic mice suggest that these types of channels are unlikely to be the mammalian auditory and vestibular mechanotransduction channels (8). Work in zebrafish has identified members of the transient receptor potential (TRP) family of ion channels as additional candidates for the mechanotransduction channel of vertebrate auditory and vestibular hair cells (9). Later work conducted with targeted deletion of the TRPA1 channel in mice, however, showed that TRP channels are not essential for hearing and vestibular functions (10).

\section{New clues provided by human genetic studies}

Despite the numerous candidates proposed up to now, the identity of the mammalian auditory and vestibular mechanotransduction channels remains elusive. A major difficulty in identifying these channels arises from the fact that there are many accessory molecules required for the mechanotransduction apparatus and they have to be properly assembled (Figure 1). Proper assembly of the mechanotransduction apparatus is a condition hard to meet in the reconstituted systems commonly used for functional verification of candidate molecules, but it is essential if functional assessments are to be made. 
Clues as to the identity of the mammalian auditory and vestibular mechanotransduction channels are also provided by the extensive data obtained from human studies of genes associated with deafness (11). Among the genes identified in such studies is TMC1. Mutations in TMC1 are associated with profound human prelingual non-syndromic deafness of both dominant (DFNA36) and recessive (DFNB7/B11) forms (12). In Iranian (13) and Turkish populations (14), TMC1 is one of the genes most commonly associated with deafness. Brownstein et al. (15) found a mutation in TMC1 to be a founder allele in the Moroccan Jewish population. For the population of Israelis of Moroccan Jewish ancestry who are deaf, recessive mutations in TMC1 were detected in more than a third of them. Consistent with these human studies linking TMC1 to an essential role in hearing, mice with Tmc1 mutations (e.g., Beethoven $[B t b]$ and deafness $[d n]$ mutant mice) generated in a large-scale $N$-ethyl- $N$ nitrosourea (ENU) mutagenesis program (16) are also deaf (17).

TMC1 and TMC2 are predicted to encode membrane proteins with six transmembrane domains. Although the specific functions of the proteins encoded by these genes are unknown, bioinformatic analysis (18) and data obtained from in vitro heterologous systems (19) suggest that they function as membrane channels or transporters (18-20). Consistent with the genetic data linking TMC1 and TMC2 to a role in hearing, it has been shown that Tmc1 and Tmc2 mRNA is expressed in the inner ear $(12,17)$. Moreover, it has been suggested that the two genes may functionally compensate for each other in inner ear functions (21).

\section{Double knockout of Tmc1 and Tmc2 reveals their roles}

In this issue of the JCI, Kawashima et al. report their novel results showing that TMC1 and TMC2 encode functionally redundant stereocilia components that are necessary for hair cell mechanotransduction in mice (4). Key to this conclusion was the observation that mice with targeted deletion of both $T m c 1$ and $T m c 2\left(T m c 1^{\Delta} T m c 2^{\Delta}\right.$ mice) showed profound deafness and vestibular dysfunction. Interestingly, mice with targeted deletion of only Tmc1 showed severe hearing loss without vestibular dysfunction, similar to what is observed in human patients with TMC1 mutation (12, 15). These results suggest that there might be functional compensation between $T m c 1$ and Tmc2. A molecular explanation for the observation was provided by quantitative RT-PCR, which showed that Tmc2 expression persisted in vestibular hair cells into adult life but was markedly reduced in cochlear hair cells after the early postnatal stage. Therefore, TMC1 was the only TMC channel expressed in the adult cochlea.

Using GFP-tagged TMC proteins to find the location of these proteins in the hair cells enabled Kawashima et al. to solve another key piece of the puzzle (4). This approach pinpointed TMC proteins to the tips of stereocilia at the apical surface of the hair cells, which is where mechanotransduction channels should be localized. High-resolution scanning electron microscopy indicated that stereocilia in hair cells from $T m c 1^{\Delta} T m c 2^{\Delta}$ mice were structurally normal, with intact tip links. However, isolated hair cells tested in vitro completely lacked mechanotransduction activity. Tmc1 and Tmc2 were then shown to be sufficient for mechanotransduction individually, since exogenous expression of either of the two genes rescued mechanotransduction activity in auditory and vestibular hair cells obtained from the $T m c 1^{\Delta} T m c 2^{\Delta}$ mice. Importantly, exogenous expression of either $T m c 1$ or $T m c 2$ rescued not only the mechanotransduction currents in the hair cells, but also uptake of fluorescent FM143 and gentamicin dye, which are both known to enter hair cells via the mechanotransduction channels. These data support the conclusion that either $T m c 1$ or $T m c 2$ is sufficient for carrying out mechanotransduction in hair cells in mice.

\section{$T m c 1^{\Delta} T m c 2^{\Delta}$ mice are valuable tools for studying deafness}

Preserving the integrity of the mammalian mechanotransduction apparatus (Figure 1) while selectively removing one piece of the jigsaw (the mechanotransduction channel) has been an insurmountable task for auditory physiologists for many years. Finally, the use of hair cells isolated from the $T m c 1^{\Delta} T m c 2^{\Delta}$ mice generated by Kawashima et al. (4) seems to provide a nearly perfect system in which the mechanotransduction channels may be the only missing piece in this complicated device (Figure 1). How the other accessory proteins (e.g., the tip links and motor proteins) interact with the mechanotransduction channels can now be tested experimentally. Where does the mechanical gate of the tip link anchor with the channels encoded by Tmc1 and Tmc2? how does stereocilia turnover (22) affect the function of mechanotransduction channels? and how do the whole mechanotransduction apparatus regenerate after chemical or noise insults? - these are just a few of the questions it is now possible to tackle with the $T m c 1^{\Delta} T m c 2^{\Delta}$ mice generated by Kawashima et al. (4). Although many aspects about the structure and functions of TMC channels still need to be answered, the work of Kawashima et al. (4) appears to have firmly established that both TMC1 and TMC2 are integral components of the mechanotransduction complex. This advances our understanding of the molecular mechanisms underlying the pathogenesis of human DFNA36, DFNB7/B11, and other types of deafness.

\section{Acknowledgments}

Research done in the author's laboratory is funded by grants from the National Institute on Deafness and Other Communication Disorders (NIDCD 4R33DC010476, R01DC010204, and R01DC006483).

Address correspondence to: Xi Lin, Departments of Otolaryngology and Cell Biology, Emory University School of Medicine, 615 Michael Street, Atlanta, Georgia 303223030, USA. Phone: 404.727.3723; Fax: 404.727.6356; E-mail: xlin2@emory.edu.

1. Hudspeth AJ. Extracellular current flow and the site of transduction by vertebrate hair cells. J Neurosci. 1982;2(1):1-10.

2. Dallos P, Santos-Sacchi J, Flock A. Intracellular recordings from cochlear outer hair cells. Science. 1982;218(4572):582-584.

3. Hille B. Sensory transduction and excitable cells. In: Hille B, ed. Ion Channels Of Excitable Membranes. Sunderland, Massachusetts, USA: Sinauer Associates, Inc.; 2001:237-268.

4. Kawashima Y, et al. Mechanotransduction in mouse inner ear hair cells requires transmembrane channellike genes. J Clin Invest. 2011;121(12):4796-4809.

5. Gullingsrud J, Kosztin D, Schulten K. Structural determinants of MscL gating studied by molecular dynamics simulations. Biophys J. 2001; 80(5):2074-2081.

6. Kolesnikov SS, Rebrik TI, Zhainazarov AB, Tavartkiladze GA, Kalamkarov GR. A cyclic-AMP-gated conductance in cochlear hair cells. FEBS Lett. 1991; 290(1-2):167-170.

7. Geffeney SL, et al. DEG/ENaC but not TRP channels are the major mechanoelectrical transduction channels in a C. elegans nociceptor. Neuron. 2011;71(5):845-857.

8. Peng BG, Ahmad S, Chen S, Chen P, Price MP, Lin X. Acid-sensing ion channel 2 contributes a major component to acid-evoked excitatory responses in spiral ganglion neurons and plays a role in noise susceptibility of mice. J Neurosci. 2004;24(45):10167-10175.

9. Corey DP, et al. TRPA1 is a candidate for the mechanosensitive transduction channel of vertebrate hair cells. Nature. 2004;432(7018):723-730.

10. Kwan KY, et al. TRPA1 contributes to cold, mechanical, and chemical nociception but is not essential for hair-cell transduction. Neuron. 2006; 
50(2):277-289.

11. Dror AA, Avraham KB. Hearing loss: mechanisms revealed by genetics and cell biology. Annu Rev Genet. 2009;43:411-437.

12. Kurima K, et al. Dominant and recessive deafness caused by mutations of a novel gene, TMC1, required for cochlear hair-cell function. Nat Genet. 2002;30(3):277-284.

13. Hildebrand MS, et al. Mutations in TMC1 are a common cause of DFNB7/11 hearing loss in the Iranian population. Ann Otol Rhinol Laryngol. 2010;119(12):830-835

14. Duman D, Sirmaci A, Cengiz FB, Ozdag H, Tekin M. Screening of 38 genes identifies mutations in $62 \%$ of families with nonsyndromic deafness in Tur- key. Genet Test Mol Biomarkers. 2011;15(1-2):29-33.

15 . Brownstein $Z$, et al. Targeted genomic capture and massively parallel sequencing to identify genes for hereditary hearing loss in middle eastern families. Genome Biol. 2011;12(9):R89.

16. Hrabe de Angelis MH, et al. Genome-wide, largescale production of mutant mice by ENU mutagenesis. Nat Genet. 2000;25(4):444-447.

17. Vreugde S, et al. Beethoven, a mouse model for dominant, progressive hearing loss DFNA36. Nat Genet. 2002;30(3):257-258.

18. Keresztes G, Mutai H, Heller S. TMC and EVER genes belong to a larger novel family, the TMC gene family encoding transmembrane proteins. BMC Genomics. 2003;4(1):24.
19. Labay V, Weichert RM, Makishima T, Griffith AJ. Topology of transmembrane channel-like gene 1 protein. Biochemistry. 2010;49(39):8592-8598.

20. Kurima K, Yang Y, Sorber K, Griffith AJ. Characterization of the transmembrane channel-like (TMC) gene family: functional clues from hearing loss and epidermodysplasia verruciformis. Genomics. 2003;82(3):300-308.

21. de Heer AM, et al. Progressive sensorineural hearing loss and normal vestibular function in a Dutch DFNB7/11 family with a novel mutation in TMC1. Audiol Neurootol. 2011;16(2):93-105.

22. Manor U, Kachar B. Dynamic length regulation of sensory stereocilia. Semin Cell Dev Biol. 2008; 19(6):502-510.

\section{Slowing down with age: lung DCs do it too}

\section{Thomas J. Braciale1,2,3 and Taeg S. Kim 1,3}

${ }^{1}$ Beirne B. Carter Center for Immunology Research, ${ }^{2}$ Department of Microbiology, and ${ }^{3}$ Department of Pathology, University of Virginia, Charlottesville, Virginia, USA.

\begin{abstract}
Decline in immune function with age has been attributed to defects or alterations in both the innate and the adaptive immune system. In this issue of the JCI, Zhao and coworkers provide evidence for a novel mechanism of immune dysfunction in aging mice. They show that migration of respiratory DCs from the site of virus replication to the draining lymph nodes in response to infection with several different respiratory viruses is markedly diminished with increasing age. The impaired DC migration was a result of increased levels of the lipid mediator prostaglandin $\mathrm{D}_{2}\left(\mathrm{PGD}_{2}\right)$ in the respiratory tract with age and could be partially reversed by blockade of $\mathrm{PGD}_{2}$ synthesis or action.
\end{abstract}

Among the many hallmarks of the aging process (in both mice and humans) is a progressive decline in immune function $(1,2)$. This is demonstrable in the diminished response of the elderly to vaccination against viral and bacterial pathogens (1). This fact compounds the more serious problem of the increased susceptibility of the elderly to infection in general and to serious and frequently life-threatening infection of the respiratory tract with respiratory viruses such as influenza A virus (IAV), respiratory syncytial virus, and severe acute respiratory syndrome coronavirus (SARS-CoV). This decline in immune function with age has been attributed to defects or alterations in both the innate and the adaptive immune system $(1,2)$. In this issue of the JCI, Zhao and coworkers provide compelling evidence for a novel mechanism of immune dysfunction in aging mice (3). They demonstrate that the migration of respiratory DCs (RDCs) from the lungs to

Conflict of interest: The authors have declared that no conflict of interest exists.

Citation for this article: J Clin Invest. 2011; 121(12):4636-4639. doi:10.1172/JCI61367. the lung draining lymph nodes (DLNs) in response to infection with several different respiratory viruses is markedly diminished with increasing age. Impaired RDC migration is mediated by the arachidonic acid (AA) metabolite prostaglandin $\mathrm{D}_{2}\left(\mathrm{PGD}_{2}\right)$, basal expression of which Zhao and coworkers show is increased in the respiratory tract with age and is further upregulated in response to infection in a pathogen-dependent fashion. They further demonstrate that blockade of either $\mathrm{PGD}_{2}$ synthesis or action partially reverses the defect in RDC migration, resulting in an enhanced adaptive immune response to infection and increased survival. These findings have interesting potential implications for both control of respiratory virus infection and vaccine efficacy in the elderly.

\section{RDC response to virus infection}

DCs are hematopoietic origin cells of the monocytic cell lineage. These cells are generally recognized to be an exclusive cell type, since they have the capacity to initiate the primary adaptive immune response (mediated by $\mathrm{T}$ and $\mathrm{B}$ cells) to foreign antigens, including infectious agents. The importance of these cells is highlighted by the fact that the 2011 Nobel Prize in Physiology or Medicine was recently awarded to Ralph Steinman for his role in the identification and characterization of DCs $(4,5)$.

DCs reside within tissues at body surfaces, such as the skin, gut, and respiratory tract mucosa. At these sites, these actively phagocytic cells act as sentinels, monitoring the site for the presence of foreign antigens, such as those derived from viruses. The tissue DCs respond to this encounter by capturing foreign antigen, processing the antigen to a form that can be recognized by $T$ cells, becoming activated, and, importantly, migrating to the DLNs from the body's surfaces. It is within the DLN that the DCs encounter T cells specific for the antigen/pathogen that they have captured and processed and, thereby, initiate the adaptive immune response (6).

Migration of activated tissue DCs from the site of infection, e.g., the lungs, to the DLN is an essential step in the induction of the adaptive immune response to the infectious agent. DC migration is chemokine regulated and, in part at least, dependent on the upregulation of expression of and signaling through the chemokine receptor CCR7, whose ligands (CCL19 and CCL21) are expressed by lymphatic endothelium draining the infected tissue (Figure 1 and ref. 7). A variety of stimuli (probably acting in concert) can stimulate tissue DC activation and, as a result, migration. These stimuli include cytokines, chemokines, and certain AA-derived metabolites, such as $\mathrm{PGE}_{2}$ and leukotriene $\mathrm{C}_{4}\left(\mathrm{LTC}_{4}\right)$ (Figure 1). Of particular relevance to the report of Zhao and coworkers is the fact that anoth- 